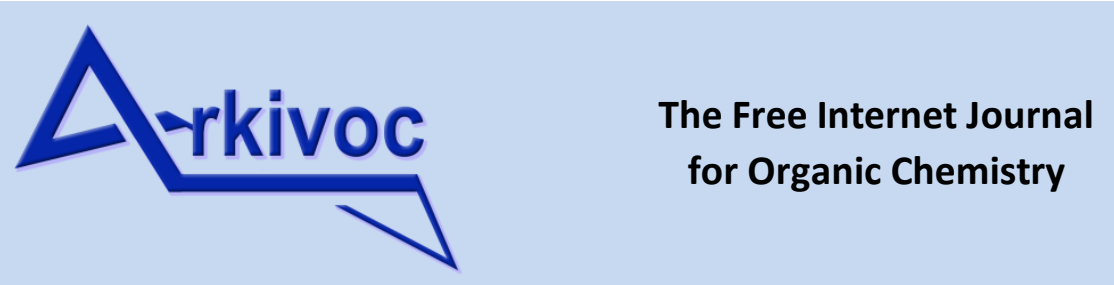

\title{
1,3,5-Triazine as core for the preparation of dendrons
}

\section{Rotimi Sheyi, ${ }^{a}$ Anamika Sharma, ${ }^{a, b}$ Ashish Kumar, ${ }^{a, b}$ Ayman El-Faham, ${ }^{c, d}$ Beatriz G. de la Torre, ${ }^{\text {b, }}$ and Fernando Albericio ${ }^{a, c, e, f^{*}}$}

${ }^{a}$ Peptide Science Laboratory, School of Chemistry and Physics, University of KwaZulu-Natal, Durban 4001, South Africa

${ }^{b}$ KwaZulu-Natal Research Innovation and Sequencing Platform (KRISP), School of Laboratory Medicine and Medical Sciences, College of Health Sciences, University of KwaZulu-Natal, Durban 4041, South Africa 'Department of Chemistry, College of Science, King Saud University, PO Box 2455, Riyadh 11451, Saudi Arabia

${ }^{d}$ Department of Chemistry, Faculty of Science, Alexandria University, PO Box 426, Alexandria 21321, Egypt

e Institute for Advanced Chemistry of Catalonia (IQAC-CSIC), 08034 Barcelona, Spain

${ }^{f}$ CIBER-BBN (Networking Centre on Bioengineering, Biomaterials and Nanomedicine) and Department of Organic Chemistry, University of Barcelona, 08028 Barcelona, Spain

*Corresponding authors.email: albericio@ukzn.ac.za; garciadelatorreb@ukzn.ac.za

\section{Abstract}

A unique property of 2,4,6-trichloro-1,3,5-triazine (TCT) is its ability to undergoes a nucleophilic aromatic substitution reaction $\left(\mathrm{S}_{N} \mathrm{Ar}\right)$ under temperature-controlled conditions. Using a convenient and biologically friendly protocol, mono-substituted s-triazines were treated with excess nucleophiles to obtain tri-substituted triazines in $1+2$ mode (one nucleophile as the first substitution followed by another nucleophile for the other two positions). The nucleophiles used for this study were phenol, thiol and amine, and the best order of incorporation was phenol or thiol for the first position followed by amine for the last two positions.

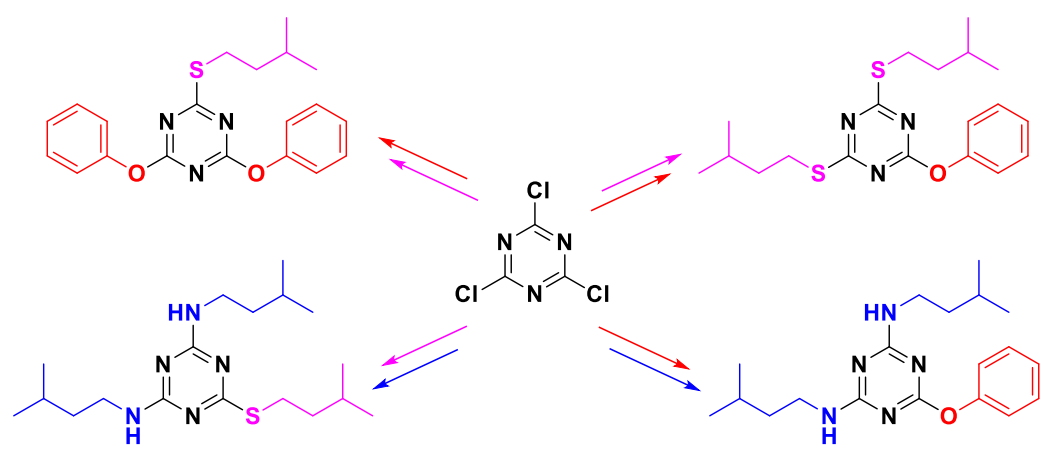

Keywords: s-Triazine, dendron, HPLC, $1+2$ mode, nucleophiles 


\section{Introduction}

s-Triazine derivatives have found numerous applications due to their potential to show biological and therapeutic activity. For example, triazine compounds have anticancer, ${ }^{1,2}{ }^{2}$ antimalarial, ${ }^{3}$ antitubercular, ${ }^{4}$ antimicrobial, ${ }^{5-9}$ and antiviral properties. ${ }^{10,11}$

2,4,6-Trichloro-1,3,5-triazine (TCT), the main reagent for the synthesis of these triazine derivatives, offers a versatile synthetic route by undergoing sequential nucleophilic substitution reactions. The ability of various nucleophiles to substitute the chlorine atoms in TCT makes this reagent suitable for the preparation of mono-, di- and trisubstituted derivatives of 1,3,5-triazine in a controlled manner. ${ }^{12-16}$

The substitution of chlorine atoms in TCT by distinct nucleophiles is a temperature-dependent sequential reaction. Thus, the general rule for the replacement of these chlorine atoms, which varies with the reactivity of the nucleophile used, proposes that the first substitution occurs at $0{ }^{\circ} \mathrm{C}$, the second at room temperature $(\mathrm{rt})$ and the third under heating conditions (Scheme 1). ${ }^{17}$
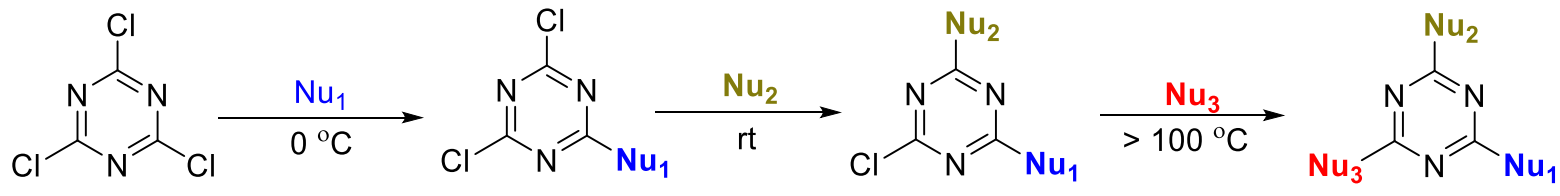

$$
\mathrm{Nu}_{1}, \mathrm{Nu}_{2}, \mathrm{Nu}_{3}=\text { Nucleophiles }
$$

Scheme 1. Nucleophilic substitution reaction for TCT.

Of note, apart from being temperature-dependent, the substitution pattern also depends on the nature of nucleophile (i.e. amines, thiols, alcohols), its basic strength, steric factors, and, more importantly, the substituents already present on the TCT/triazine (TA) core. For instance, the presence of an amine nucleophile on the TCT/TA core prevents the incorporation of nucleophiles other than amines. ${ }^{18-20}$

TCT chemistry also encompasses orthogonal chemoselective properties, two interchangeable concepts used in chemistry. The concept of orthogonality was first introduced in 1977 by Barany and Merrifield ${ }^{21}$ to explain an orthogonal protecting scheme, and it was later demonstrated by Barany and Albericio in 1985 in a sequential triorthogonal protection scheme. ${ }^{22}$ Chemoselectivity, as used by Trost in $1983^{23}$, is the ability of a chemical reagent to discriminate among reactive sites. Hence, in practice, the concept of orthogonal chemoselectivity is the discrimination between reactive sites in any order in different chemical mechanisms. ${ }^{18}$

TCT has also found application in the synthesis of dendrimers. ${ }^{24,}{ }^{25}$ The term "dendrimer" comes from the Greek word meaning "tree". In chemistry, dendrimers are repetitive branching molecules, formed by a nucleus (core) molecule, branching units, branches, and peripheral groups, which could be the main responsible for the biological activity. ${ }^{26-29}$ In 1985, Tomalia and co-workers introduced the concept of a dendrimer with the idea of it being used in many applications, ranging from catalysis to electronics and drug delivery. ${ }^{30,31}$ As part of a dendrimer, the ramified branches without the nucleus is known as a dendron.

In previous work, we studied the orthogonal chemoselective property of TCT using three nucleophiles (phenol, 3-methylbutane-1-thiol and 3-methylbutan-1-amine). ${ }^{19}$ The best order and condition for the incorporation of these nucleophiles was found to be phenol $(\mathrm{O})$ at $0{ }^{\circ} \mathrm{C}$, thiol $(\mathrm{S})$ at $\mathrm{rt}$, and finally amine $(\mathrm{N})$ at $35{ }^{\circ} \mathrm{C}$, the latter temperature compatible with biological systems. ${ }^{19}$ The incorporation was achieved through the electron-withdrawing behavior of phenol, which in turn deactivates the ring, hence maintaining electron 
delocalization for further incorporation of thiol or amine at ambient temperature. The nucleophiles chosen are to mimic the reactivity of Lys for amine, Tyr for phenol and Cys for thiol.

With the successful replacement of the third chlorine at $35^{\circ} \mathrm{C}$ and taking advantage of the reactivity of the TCT chlorine atoms into consideration, this study sought to test the ability of TCT to demonstrate $1+2$ mode where the core is first substituted with a nucleophile to afford mono-substituted derivative which with excess of another nucleophile (different from the first one incorporated onto mono-substituted triazine) to give trisubstituted triazine molecules under, if possible, ambient condition.

\section{Results and Discussion}

Scheme 2 shows the synthetic route used to obtain compounds 1-9. Using TCT as the starting material, the derivatives were synthesized by sequentially substituting its chlorine atoms with an equivalent amount of the three different nucleophile(s). As a solvent, ethyl acetate was used due to its higher boiling point in comparison with $\mathrm{CH}_{2} \mathrm{Cl}_{2}$, which was used in previous work. ${ }^{18}$

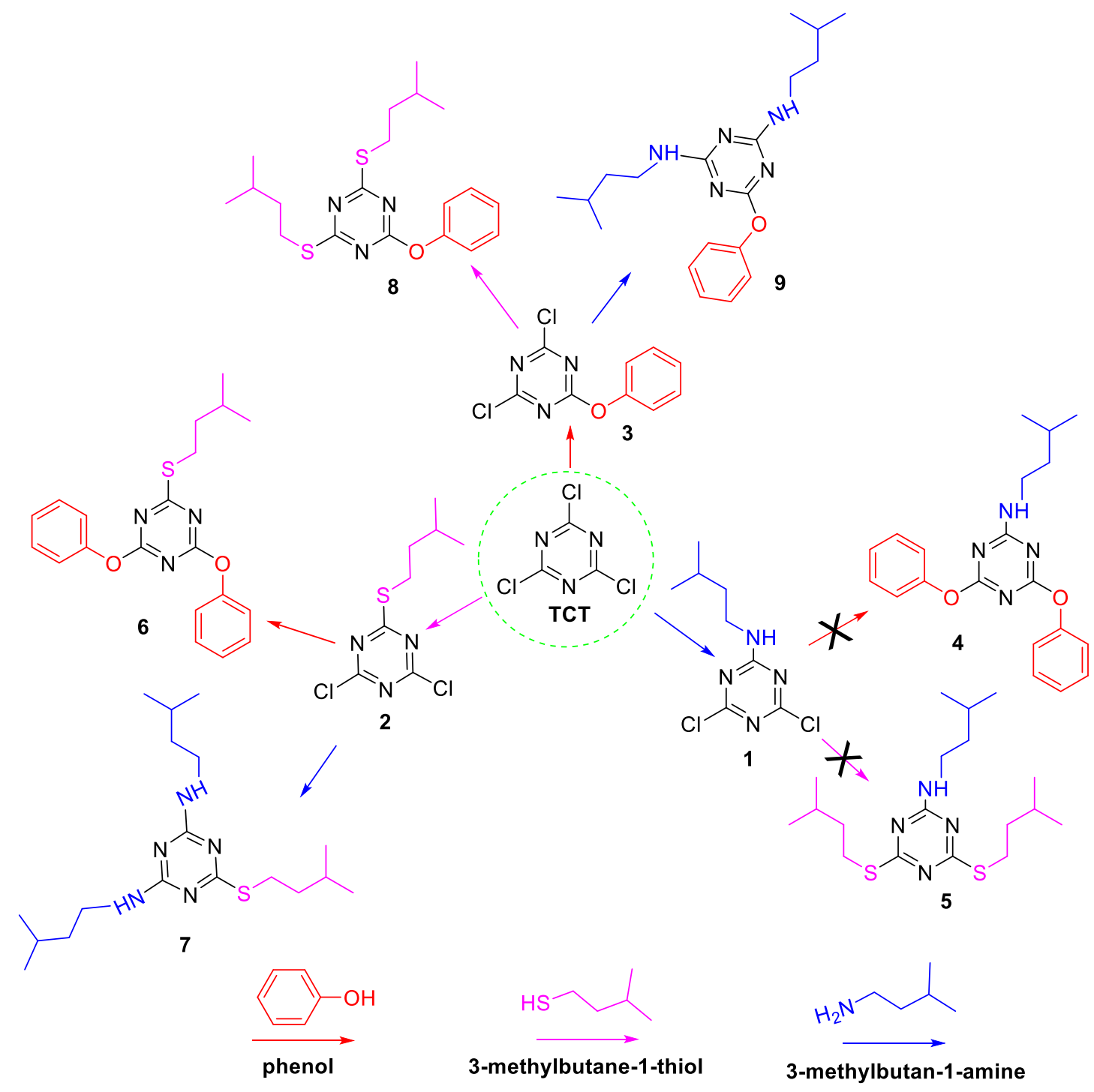

Scheme 2. Synthetic route for obtaining compounds 1-9. 
Mono-substituted triazine derivatives were prepared by reacting 1 equivalent of nucleophile (thiol, amine and phenol) with 1 equivalent of TCT at $0{ }^{\circ} \mathrm{C}$ (except phenol, which was achieved at $-20{ }^{\circ} \mathrm{C}$, a condition validating our previous work) ${ }^{19}$ in the presence of diisopropylethylenamine (DIEA) as base, as reported earlier by our group. ${ }^{18-20}$ The reaction was completed in $30 \mathrm{~min}$, as monitored by TLC, affording compounds 1-3 (Figure 1).

With the successful synthesis of mono-substituted triazine, 2 equivalents of each successive nucleophile were added in each case (1-3). For 1, no product formation was observed. This result is consistent with our earlier findings showing that once an amine is incorporated, only another amine can be incorporated under the given reaction conditions. ${ }^{18,19}$ Therefore, $\mathbf{4}$ and $\mathbf{5}$ could not be prepared via this route.

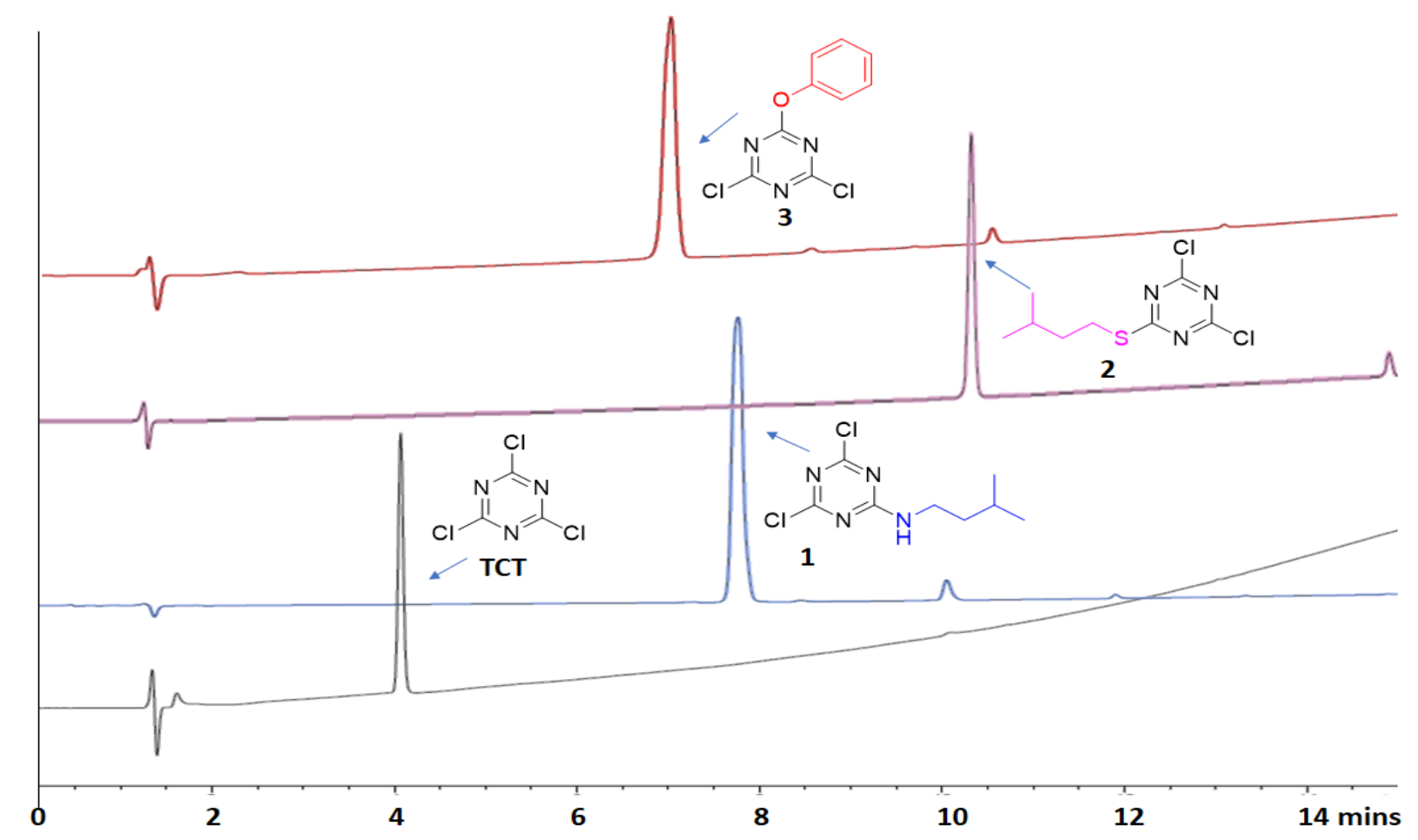

Figure 1. HPLC chromatogram of compounds 1,2 and 3.

Similarly, $\mathbf{2}$ was treated with two equivalents of amine, and phenol nucleophiles were added in parallel, forming 6 and 7. At first, the reactions were allowed to stir at room temperature for $3 \mathrm{~h}$. New products were formed; however, TLC showed that the reactions were not complete, so they were kept for $12 \mathrm{~h}$ at $35^{\circ} \mathrm{C}$. TLC showed the complete consumption of the starting material, affording the product (6 and 7), as monitored by HPLC (Figure 2). 


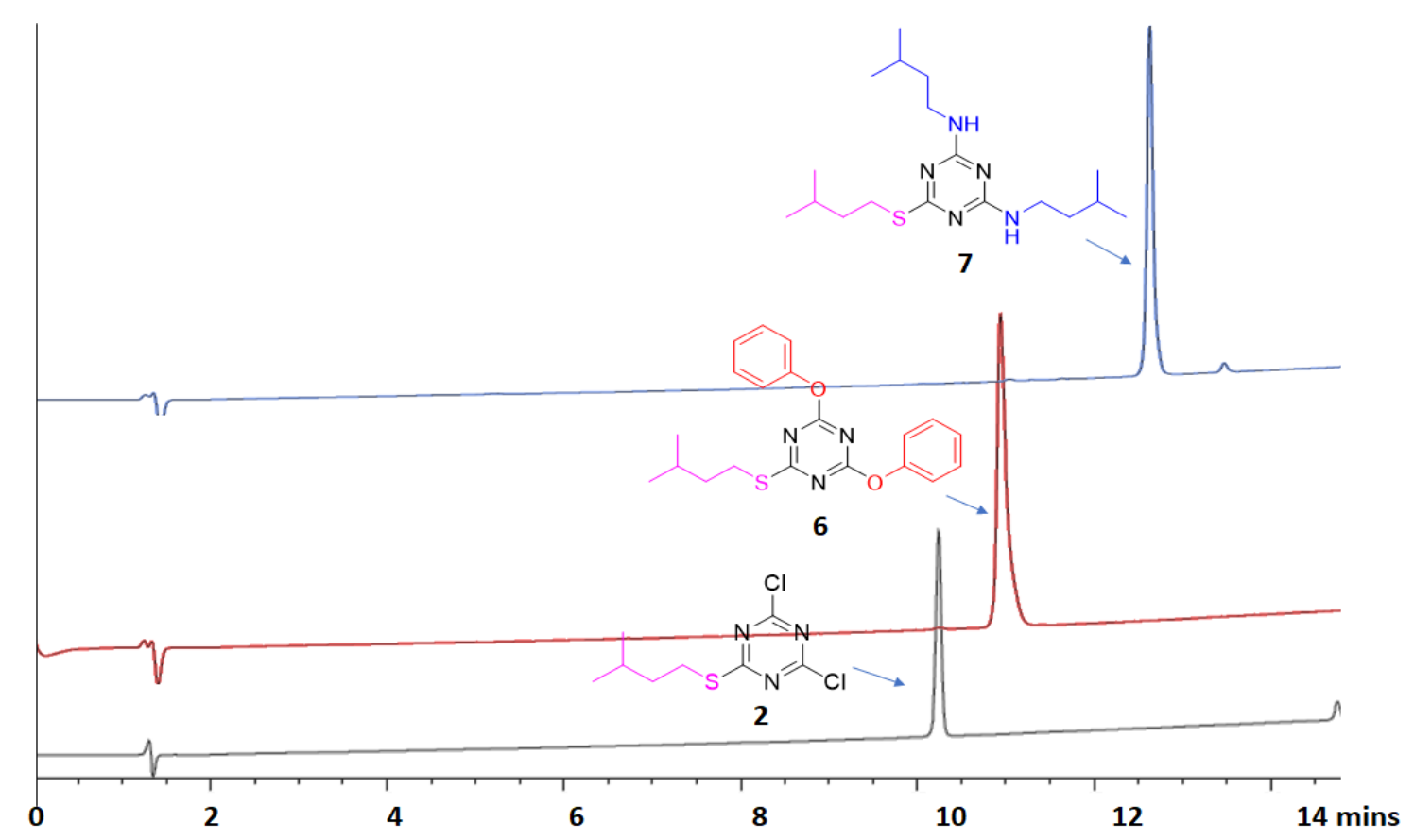

Figure 2. Chromatogram of compounds 6 and 7, as monitored by HPLC.

In parallel, $\mathbf{3}$ was used to synthesize $\mathbf{8}$ and $\mathbf{9}$ by adding 2 equivalents of thiol and amine nucleophiles followed by the addition of 2 equivalents of DIEA. The reaction was kept at $35^{\circ} \mathrm{C}$ for $12 \mathrm{~h}$. Completion of the reaction was monitored by TLC, affording 8 and 9 (Figure 3).

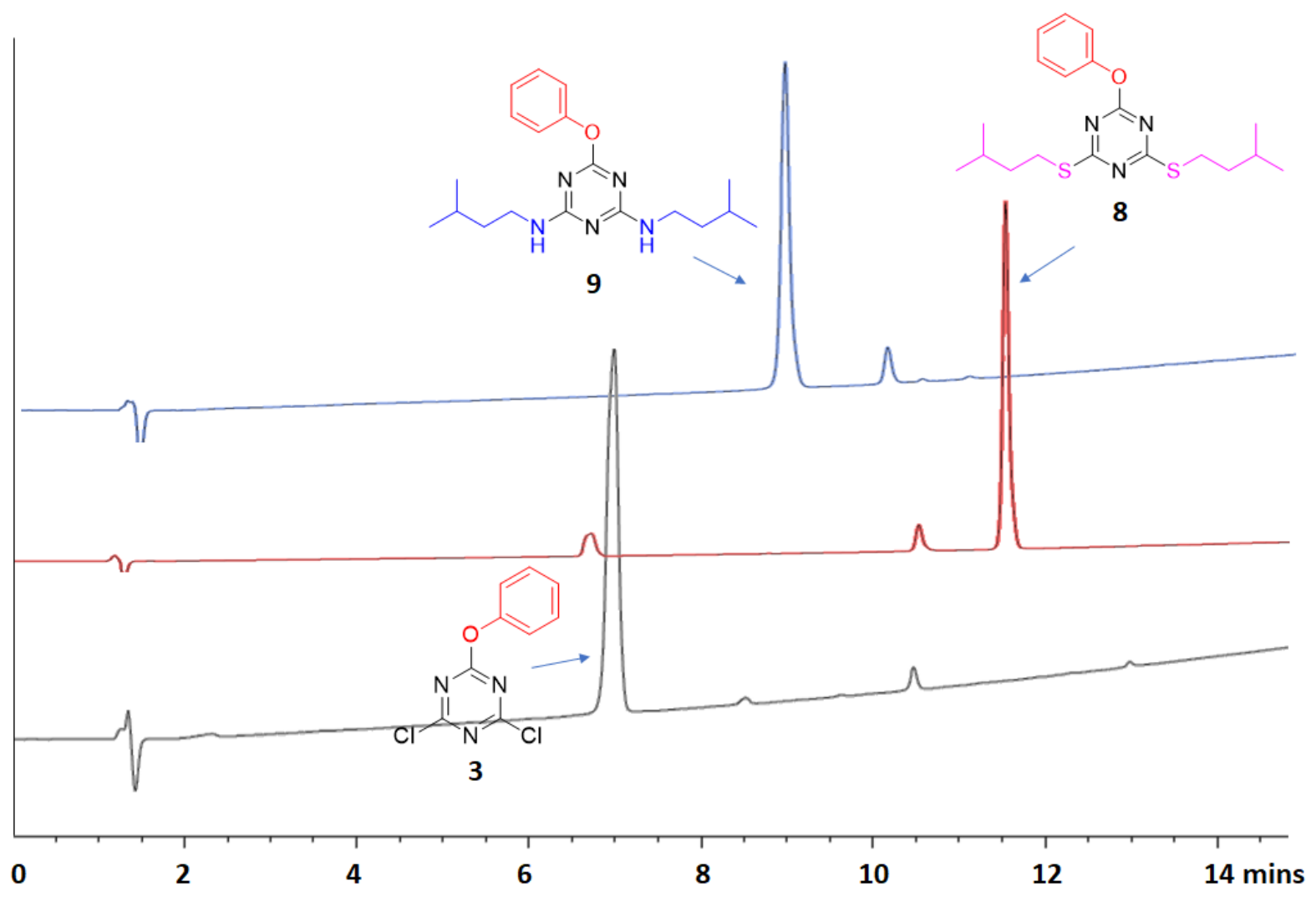

Figure 3. Chromatogram of compounds 8 and 9 , as monitored by HPLC. 


\section{Conclusions}

In conclusion, here we report the preparation of triazine derivatives/dendrons by sequential incorporation of nucleophiles (phenol, amine and thiol) onto TCT. To this end, 2 equivalents of these nucleophiles was added to a mono-substituted triazine at $35^{\circ} \mathrm{C}$, a temperature compatible with biological systems. The strategy works in cases where thiol and phenol are first substituents. However, once amine is incorporated, no reaction with other nucleophiles occurs. Replacement of the third chlorine atom at $35{ }^{\circ} \mathrm{C}$ extends the use of TCT beyond a triorthogonal linker in the biological context, thereby paving the way for nucleophilic reactions involving various peptides, antibodies, and drugs. The applications of this strategy for the preparation of dendrimers of biological interest using Lys, Tyr and Cys amino acids bearing peptides are currently being carried out in our laboratories and will be described elsewhere.

\section{Experimental Section}

General. HPLC-grade solvents were used for all the synthetic preparations. 2,4,6-Trichloro-1,3,5-triazine (TCT), isopentylamine, 3-methylbutane-1-thiol, phenol, and diisopropylethylamine were purchased from SigmaAldrich (Sigma-Aldrich, Germany). Magnetic resonance spectra $\left({ }^{1} \mathrm{H}\right.$ NMR and ${ }^{13} \mathrm{C}$ NMR) were recorded on a Bruker $400 \mathrm{MHz}$, and chemical shift values are reported in $\delta$ units (ppm) using TMS as internal standard ( $\delta$ 0.00 ) and $\mathrm{CDCl}_{3}$ as solvent $\left(\delta 7.2\right.$ for ${ }^{1} \mathrm{H}$ and $\delta 77.20$ for ${ }^{13} \mathrm{C}$ ). Follow-up of the reactions and checks of the purity of the compound were done by TLC on silica-gel-protected aluminium sheets $60 \mathrm{~F} 254$ (Merck), and the spots were detected by exposure to UV light at $\lambda 254 \mathrm{~nm}$. Analytical HPLC was performed on an Agilent 1100 system using a Phenomenex $\mathrm{C}_{18}$ column $(3 \mu \mathrm{m}, 4.6 \times 50 \mathrm{~mm})$ by dissolving the sample in $\mathrm{CH}_{3} \mathrm{CN}$ only. Chemstation software was used for data processing. Buffer A: $0.1 \%$ TFA in $\mathrm{H}_{2} \mathrm{O}$, buffer $\mathrm{B}: 0.1 \%$ TFA in $\mathrm{CH}_{3} \mathrm{CN}$ were used in HPLC. High-resolution mass spectrometry (HRMS) was performed using a Bruker ESI-QTOF mass spectrometer in positive-ion mode.

Synthesis of 2,4-dichloro-6-substituted s-triazine (X-DCT)

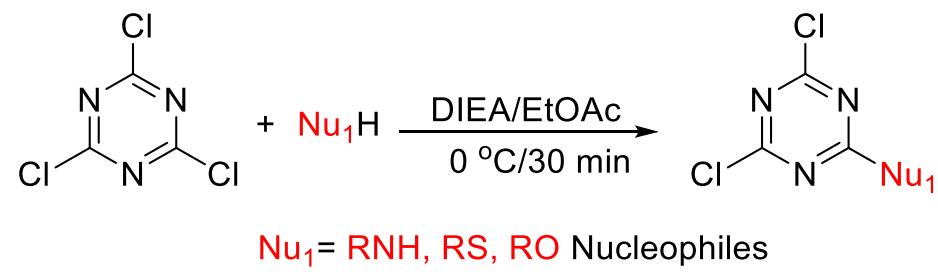

Scheme 3. Synthetic scheme for the formation of mono-substituted triazine.

TCT (50 mg, $0.27 \mathrm{mmol})$ was dissolved in EtOAc $(1 \mathrm{~mL})$ and cooled to $0{ }^{\circ} \mathrm{C}$ for $5 \mathrm{~min}$. Nucleophile $(0.27 \mathrm{mmol}$; $31.3 \mu \mathrm{L}$ amine or $33.7 \mu \mathrm{L}$ thiol or $25.4 \mathrm{mg}$ phenol) was then added to the above stirring solution, followed by addition of DIEA ( $47 \mu \mathrm{L}, 0.27 \mathrm{mmol})$. The reaction was stirred at $0{ }^{\circ} \mathrm{C}$ for $30 \mathrm{~min}$. The progress of the reaction was monitored by TLC (EtOAC/hexane as mobile phase) until no starting material was observed. The reaction mixture was diluted with DCM $(5 \mathrm{~mL})$ and washed several times with water to remove DIEA salts. The organic layer was collected, dried over $\mathrm{MgSO}_{4}$, filtered, and concentrated to afford the pure product, which was used for the next step without further purification. 
4,6-Dichloro- $\mathbf{N}$-isopentyl-1,3,5-triazin-2-amine (1). Off-white semi-solid, 94\% yield; HPLC [30-95 \% of $\mathrm{CH}_{3} \mathrm{CN}$ (0.1\% TFA/ $\mathrm{H}_{2} \mathrm{O}(0.1 \%$ TFA) over $15 \mathrm{~min}] t_{R} 7.7 \mathrm{~min} ;{ }^{1} \mathrm{H} \mathrm{NMR}\left(400 \mathrm{MHz}, \mathrm{CDCl}_{3}\right): 0.88$ (d, J $\left.6.6 \mathrm{~Hz}, 6 \mathrm{H},-\mathrm{CH}_{3}\right), 1.43$ (q, J $\left.7.5 \mathrm{~Hz}, 2 \mathrm{H},-\mathrm{CH}_{2}\right), 1.59(\mathrm{~m}, 1 \mathrm{H}, \mathrm{CH}), 3.42\left(\mathrm{~m}, 2 \mathrm{H},-\mathrm{CH}_{2}\right), 5.70$ (brs, NH); ${ }^{13} \mathrm{C} \mathrm{NMR}\left(100 \mathrm{MHz}, \mathrm{CDCl}_{3}\right): 22.4$, 25.7, 37.9, 39.9, 165.8, 171.0; LCMS m/z: calcd. for $\mathrm{C}_{8} \mathrm{H}_{12} \mathrm{Cl}_{2} \mathrm{~N}_{4}$ : 234.0439; found: 235.1224 [M+H] ${ }^{+}, 278.1577$ $\left[\mathrm{M}+\mathrm{CH}_{3} \mathrm{CN}+\mathrm{H}\right]^{+}$.

2,4-Dichloro-6-(isopentylthio)-1,3,5-triazine (2). Yellowish oil, 92\% yield; HPLC [30-95\% of $\mathrm{CH}_{3} \mathrm{CN}(0.1 \% \mathrm{TFA} /$ $\mathrm{H}_{2} \mathrm{O}(0.1 \% T F A)$ over $\left.15 \mathrm{~min}\right] t_{R} 10.2 \mathrm{~min} ;{ }^{1} \mathrm{H} \mathrm{NMR}\left(400 \mathrm{MHz}, \mathrm{CDCl}_{3}\right): 0.90\left(\mathrm{~d}, J 6.5 \mathrm{~Hz}, 6 \mathrm{H},-\mathrm{CH}_{3}\right), 1.54(\mathrm{~m}, 2 \mathrm{H}$, $\left.\mathrm{CH}_{2}\right), 1.65(\mathrm{~m}, 1 \mathrm{H},-\mathrm{CH}), 3.10\left(\mathrm{~m}, 2 \mathrm{H}, \mathrm{CH}_{2}\right) ;{ }^{13} \mathrm{C} \mathrm{NMR}\left(100 \mathrm{MHz}, \mathrm{CDCl}_{3}\right): 21.1,26.4,28.3,36.2,168.9,185.6$. HRMS: $m / z$ : calcd. for $\mathrm{C}_{8} \mathrm{H}_{11} \mathrm{Cl}_{2} \mathrm{~N}_{3} \mathrm{~S}: 252.0251[\mathrm{M}+\mathrm{H}]^{+}$; found: 252.0246 .

2,4-Dichloro-6-phenoxy-1,3,5-triazine (3). Off-white solid, 95\% yield; HPLC [30-95\% of $\mathrm{CH}_{3} \mathrm{CN}\left(0.1 \%\right.$ TFA/ $\mathrm{H}_{2} \mathrm{O}$ (0.1\%TFA) over $15 \mathrm{~min}] t_{R} 6.9 \mathrm{~min} ;{ }^{1} \mathrm{H}$ NMR $\left(400 \mathrm{MHz}, \mathrm{CDCl}_{3}\right): 7.14(\mathrm{~d}, J 8.1 \mathrm{~Hz}, 2 \mathrm{H}, \mathrm{Ar}-\mathrm{H}), 7.26(\mathrm{t}, J 7.4 \mathrm{~Hz}, 1 \mathrm{H}$, $\mathrm{Ar}-\mathrm{H}), 7.38$ (t, J $7.8 \mathrm{~Hz}, 1 \mathrm{H}, \mathrm{Ar}-\mathrm{H}) ;{ }^{13} \mathrm{C}$ NMR (100 MHz, $\left.\mathrm{CDCl}_{3}\right): 121.2,126.5,129.7,151.3,172.4,173.8$. HRMS: $\mathrm{m} / \mathrm{z}$ : calcd. for $\mathrm{C}_{9} \mathrm{H}_{5} \mathrm{Cl}_{2} \mathrm{~N}_{3} \mathrm{O}: 241.0904[\mathrm{M}+\mathrm{H}]^{+}$; found: 241.0886.

Synthesis of 2,4,6-trisubstituted s-triazine (XYY-T)

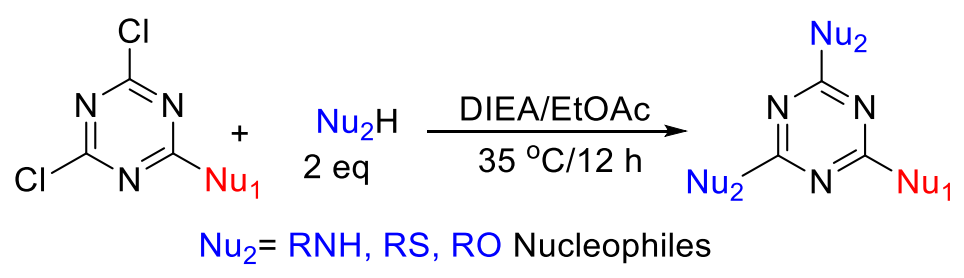

Scheme 4. Synthetic scheme for the formation of tri-substituted-triazine.

The corresponding nucleophile ( 2 equivalents, $0.54 \mathrm{mmol} ; 62.6 \mu \mathrm{L}$ amine or $67.4 \mu \mathrm{L}$ thiol or $50.8 \mathrm{mg}$ phenol) was added to the mono-substituted triazine (X-DCT) $(0.27 \mathrm{mmol})$ in EtOAc $(1 \mathrm{~mL})$, followed by addition of DIEA $(94 \mu \mathrm{L}, 0.54 \mathrm{mmol})$. The reaction was stirred at $35^{\circ} \mathrm{C}$ for $12 \mathrm{~h}$. The progress of the reaction was monitored by TLC (EtOAc/hexane) until no starting material was observed. The reaction mixture was diluted with DCM (5 $\mathrm{mL}$ ) and washed several times with water to remove DIEA salts. The organic layer was collected, dried over $\mathrm{MgSO}_{4}$, filtered, and concentrated to afford the pure product.

2-(Isopentylthio)-4,6-diphenoxy-1,3,5-triazine (6). The target compound was prepared from 2 . Yellowish semi solid, $90 \%$ yield; $\mathrm{HPLC}$ [30-95\% of $\mathrm{CH}_{3} \mathrm{CN}\left(0.1 \% \mathrm{TFA} / \mathrm{H}_{2} \mathrm{O}(0.1 \% \mathrm{TFA})\right.$ over $15 \mathrm{~min}$ ] $t_{R} 10.9 \mathrm{~min} ;{ }^{1} \mathrm{H} \mathrm{NMR}(400$ $\left.\mathrm{MHz}, \mathrm{CDCl}_{3}\right): 0.72\left(\mathrm{~d}, \mathrm{~J} 6.4 \mathrm{~Hz}, 6 \mathrm{H},-\mathrm{CH}_{3}\right), 1.35\left(\mathrm{~m}, 2 \mathrm{H},-\mathrm{CH}_{2}\right), 1.38(\mathrm{~m}, 1 \mathrm{H},-\mathrm{CH}), 2.81\left(\mathrm{~m}, 2 \mathrm{H},-\mathrm{CH}_{2}\right), 7.08(\mathrm{~m}, 4 \mathrm{H}$, $\operatorname{Ar}-\mathrm{H}), 7.16(\mathrm{~m}, 2 \mathrm{H}, \mathrm{Ar}-\mathrm{H}), 7.30(\mathrm{~m}, 4 \mathrm{H}, \mathrm{Ar}-\mathrm{H}) ;{ }^{13} \mathrm{C} \mathrm{NMR}\left(100 \mathrm{MHz}, \mathrm{CDCl}_{3}\right): 22.2,27.5,28.5,37.9,121.7,125.8$, 129.4, 151.8, 167.9, 183.3. HRMS: $\mathrm{m} / \mathrm{z}$ : calcd. for $\mathrm{C}_{20} \mathrm{H}_{21} \mathrm{~N}_{3} \mathrm{O}_{2} \mathrm{~S}: 368.1433[\mathrm{M}+\mathrm{H}]^{+}$; found: 368.1745 .

$\mathbf{N}^{2}, \mathbf{N}^{4}$-Diisopentyl-6-(isopentylthio)-1,3,5-triazine-2,4-diamine (7). The target compound was prepared from 2. Creamy solid, $88 \%$ yield; $\mathrm{HPLC}\left[30-95 \%\right.$ of $\mathrm{CH}_{3} \mathrm{CN}\left(0.1 \% \mathrm{TFA} / \mathrm{H}_{2} \mathrm{O}(0.1 \% \mathrm{TFA})\right.$ over $\left.15 \mathrm{~min}\right] t_{R} 12.6 \mathrm{~min}$; ${ }^{1} \mathrm{H}$ NMR $\left(400 \mathrm{MHz}, \mathrm{CDCl}_{3}\right): 0.90\left(\mathrm{~m}, 18 \mathrm{H},-\mathrm{CH}_{3}\right), 1.46\left(\mathrm{~m}, 6 \mathrm{H},-\mathrm{CH}_{2}\right), 1.65(\mathrm{~m}, 3 \mathrm{H},-\mathrm{CH}), 3.00\left(\mathrm{~m}, 4 \mathrm{H},-\mathrm{CH}_{2}\right), 3.36(\mathrm{~m}$, $\left.2 \mathrm{H},-\mathrm{CH}_{2}\right), 5.26$ (brs, $\left.\mathrm{NH}\right) ;{ }^{13} \mathrm{C} \mathrm{NMR}\left(100 \mathrm{MHz} \mathrm{CDCl}_{3}\right): 21.2,21.4,24.8,26.4,26.6,27.6,36.9,38.6,163.1,167.0$, 182.5. HRMS: $m / z$ : calcd. for $\mathrm{C}_{18} \mathrm{H}_{35} \mathrm{~N}_{5} \mathrm{~S}: 354.2691[\mathrm{M}+\mathrm{H}]^{+}$; found: 354.3031.

2,4-Bis(isopentylthio)-6-phenoxy-1,3,5-triazine (8). The target compound was prepared from 3. Pale yellow oil, 92\% yield; HPLC [30-95\% of $\mathrm{CH}_{3} \mathrm{CN}\left(0.1 \% \mathrm{TFA} / \mathrm{H}_{2} \mathrm{O}(0.1 \% \mathrm{TFA})\right.$ over $15 \mathrm{~min}$ ] $t_{R} 11.6 \mathrm{~min} ;{ }^{1} \mathrm{H} \mathrm{NMR}(400 \mathrm{MHz}$, $\left.\mathrm{CDCl}_{3}\right)$ : $0.77\left(\mathrm{~d}, \mathrm{~J} 6.5 \mathrm{~Hz}, 12 \mathrm{H},-\mathrm{CH}_{3}\right), 1.40\left(\mathrm{~m}, 4 \mathrm{H}, \mathrm{CH}_{2}\right), 1.50(\mathrm{~m}, 2 \mathrm{H},-\mathrm{CH}), 2.90\left(\mathrm{~m}, 4 \mathrm{H},-\mathrm{CH}_{2}\right), 7.08(\mathrm{~m}, 2 \mathrm{H}, \mathrm{Ar}-$ $\mathrm{H}), 7.21(\mathrm{~m}, 1 \mathrm{H}, \mathrm{Ar}-\mathrm{H}), 7.31(\mathrm{~m}, 2 \mathrm{H}, \mathrm{Ar}-\mathrm{H}) ;{ }^{13} \mathrm{C} \mathrm{NMR}\left(100 \mathrm{MHz}, \mathrm{CDCl}_{3}\right): 21.1,26.4,27.9,36.6,120.3,125.3$, 128.6, 150.5, 169.9, 185.6. LCMS: $\mathrm{m} / z$ : calcd. for $\mathrm{C}_{19} \mathrm{H}_{27} \mathrm{~N}_{3} \mathrm{OS}_{2}: 377.1596[\mathrm{M}+\mathrm{H}]^{+}$; found: 378.2227 . 
$\mathbf{N}^{2}, \mathbf{N}^{\mathbf{4}}$-Diisopentyl-6-phenoxy-1,3,5-triazine-2,4-diamine (9). The target compound was prepared from 3. Offwhite solid, 90\% yield; $\mathrm{HPLC}\left[30-95 \%\right.$ of $\mathrm{CH}_{3} \mathrm{CN}\left(0.1 \% \mathrm{TFA} / \mathrm{H}_{2} \mathrm{O}(0.1 \% \mathrm{TFA})\right.$ over $\left.15 \mathrm{~min}\right] t_{R} 8.9 \mathrm{~min} ;{ }^{1} \mathrm{H} \mathrm{NMR}$ (400 MHz, CDCl $)$ : $0.91\left(\mathrm{~m}, 12 \mathrm{H},-\mathrm{CH}_{3}\right), 1.26(\mathrm{~m}, 2 \mathrm{H},-\mathrm{CH}), 1.44\left(\mathrm{~m}, 4 \mathrm{H},-\mathrm{CH}_{2}\right), 3.41\left(\mathrm{~m}, 4 \mathrm{H},-\mathrm{CH}_{2}\right), 4.93(\mathrm{brs},-\mathrm{NH})$, 5.10 (brs, NH), 7.12 (m, 3H, Ar-H), 7.36 (m, 2H, Ar-H); $\left.{ }^{13} \mathrm{C} \mathrm{NMR} \mathrm{(100} \mathrm{MHz,} \mathrm{CDCl}_{3}\right): 21.3,24.6,36.9,38.6,120.6$, $124.8,128.3,150.9,166.0,169.8$. HRMS: $m / z$ : calcd. for $\mathrm{C}_{19} \mathrm{H}_{29} \mathrm{~N}_{5} \mathrm{O}: 344.2450[\mathrm{M}+\mathrm{H}]^{+}$; found: 344.2819 .

\section{Acknowledgements}

The work was funded by: Researchers Supporting Project at King Saud University (RSP-2020/50) (Saudi Arabia); National Research Foundation (NRF) and the University of KwaZulu-Natal (South Africa); the Spanish Ministry of Economy, Industry, and Competitiveness (MINECO) (RTI2018-093831-B-100), CIBER-BBN, and the Generalitat de Catalunya (2017 SGR 1439) (Spain). Rotimi Sheyi thanks to MINTEK for a predoctoral fellowship.

\section{Supplementary Material}

The supplementary information includes copies of HPLC, ${ }^{1} \mathrm{H}$ NMR and ${ }^{13} \mathrm{C}$ NMR spectra and HRMS.

\section{References}

1. Kumar, R.; Gupta, L.; Pal, P.; Khan, S.; Singh, N.; Katiyar, S. B.; Meena, S.; Sarkar, J.; Sinha, S.; Kanaujiya, J. K.; Lochab, S.; Trivedi, A. K.; Chauhan, P. M. S. Eur. J. Med. Chem. 2010, 45, 2265-2276. https://doi.org/10.1016/j.ejmech.2010.02.001

2. Menicagli, R.; Samaritani, S.; Signore, G.; Vaglini, F.; Dalla Via, L. J. Med. Chem. 2004, 47, 4649-4652. https://doi.org/10.1021/jm0495374

3. Kumar, A.; Srivastava, K.; Raja Kumar, S.; Puri, S. K.; Chauhan, P. M. S. Bioorg. Med. Chem. Lett. 2008, 18, 6530-6533.

https://doi.org/10.1016/j.bmcl.2008.10.049

4. Patel, A. B.; Chikhalia, K. H.; Kumari, P. Eur. J. Med. Chem. 2014, 79, 57-65.

https://doi.org/10.1016/i.ejmech.2014.03.085

5. Desai, N. C.; Makwana, A. H.; Rajpara, K. M. J. Saudi Chem. Soc. 2016, 20, S334-S341. https://doi.org/10.1016/i.jscs.2012.12.004

6. Gahtori, P.; Ghosh, S. K.; Singh, B.; Singh, U. P.; Bhat, H. R.; Uppal, A. Saudi Pharm. J. 2012, 20, 35-43. https://doi.org/10.1016/j.jsps.2011.05.003

7. Ma, X.; Tan, S.-T.; Khoo, C.-L.; Sim, H.-M.; Chan, L.-W.; Chui, W.-K. Bioorg. Med. Chem. Lett. 2011, 21, 54285431.

https://doi.org/10.1016/j.bmcl.2011.06.125

8. Saleh, M.; Abbott, S.; Perron, V.; Lauzon, C.; Penney, C.; Zacharie, B. Bioorg. Med. Chem. Lett. 2010, 20, 945-949. https://doi.org/10.1016/j.bmcl.2009.12.063

9. Singh, R. B.; Das, N.; Zaman, M. Int. J. Med. Chem. 2015, 2015, 1-10. https://doi.org/10.1155/2015/571836 
10. Dao, P.; Jarray, R.; Le Coq, J.; Lietha, D.; Loukaci, A.; Lepelletier, Y.; Hadj-Slimane, R.; Garbay, C.; Raynaud, F.; Chen, H. Bioorg. Med. Chem. Lett. 2013, 23, 4552-4556.

https://doi.org/10.1016/i.bmcl.2013.06.038

11. Wang, Q.; Liu, G.; Shao, R.; Huang, R. Heteroatom Chem. 2003, 14, 542-545. https://doi.org/10.1002/hc.10189

12. Arduini, M.; Crego-Calama, M.; Timmerman, P.; Reinhoudt, D. N. J. Org. Chem. 2003, 68, 1097-1106. https://doi.org/10.1021/jo020610c

13. de Hoog, P.; Gamez, P.; Driessen, W. L.; Reedijk, J. Tetrahedron Lett. 2002, 43, 6783-6786. https://doi.org/10.1016/S0040-4039(02)01498-3

14. Hayashi, T.; Fujimoto, A.; Kajiki, T.; Kondo, S.-i.; Yano, Y. Chem. Lett. 2000, 29, 1018-1019. https://doi.org/10.1246/cl.2000.1018

15. Saha, B. K.; Jetti, R. K. R.; Reddy, L. S.; Aitipamula, S.; Nangia, A. Cryst. Growth Des. 2005, 5, 887-899. https://doi.org/10.1021/cg049691r

16. Silen, J. L.; Lu, A. T.; Solas, D. W.; Gore, M. A.; Maclean, D.; Shah, N. H.; Coffin, J. M.; Bhinderwala, N. S.; Wang, Y.; Tsutsui, K. T.; Look, G. C.; Campbell, D. A.; Hale, R. L.; Navre, M.; DeLuca-Flaherty, C. R. Antimicrob. Agents Chemother. 1998, 42, 1447.

https://doi.org/10.1128/AAC.42.6.1447

17. Blotny, G. Tetrahedron 2006, 62, 9507-9522. https://doi.org/10.1016/j.tet.2006.07.039

18. Sharma, A.; El-Faham, A.; de la Torre, B. G.; Albericio, F. Front. Chem. 2018, 6, 1-11. https://doi.org/10.3389/fchem.2018.00516

19. Sheyi, R.; Sharma, A.; El-Faham, A.; de la Torre, B. G.; Albericio, F. Aust. J. Chem. 2020, 73, 352-356. https://doi.org/10.1071/CH19524

20. Sharma, A.; Sheyi, R.; Kumar, A.; El-Faham, A.; de la Torre, B. G.; Albericio, F. Org. Lett. 2019, 21, 78887892. https://doi.org/10.1021/acs.orglett.9b02878

21. Barany, G.; Merrifield, R. B. J. Am. Chem. Soc. 1977, 99, 7363-7365. https://doi.org/10.1021/ja00464a050

22. Barany, G.; Albericio, F. J. Am. Chem. Soc. 1985, 107, 4936-4942. https://doi.org/10.1021/ja00303a019

23. Trost, B. M. Science 1983, 219, 245-250. https://doi.org/10.1126/science.219.4582.245

24. Steffensen, M. B.; Hollink, E.; Kuschel, F.; Bauer, M.; Simanek, E. E. J. Polym. Sci. A: Polym. Chem. 2006, 44, 3411-3433. https://doi.org/10.1002/pola.21333

25. Lim, J.; Simanek, E. E. Adv. Drug Deliv. Rev. 2012, 64, 826-835. https://doi.org/10.1016/j.addr.2012.03.008

26. Fréchet, J. M. J. J. Polym. Sci. A: Polym. Chem. 2003, 41, 3713-3725. https://doi.org/10.1002/pola.10952

27. Grayson, S. M.; Fréchet, J. M. J. Chem. Rev. 2001, 101, 3819-3868. https://doi.org/10.1021/cr990116h

28. Lee, C. C.; MacKay, J. A.; Fréchet, J. M. J.; Szoka, F. C. Nat. Biotechnol. 2005, 23, 1517-1526. https://doi.org/10.1038/nbt1171

29. Tomalia, D. A.; Fréchet, J. M. J. J. Polym. Sci. A: Polym. Chem. 2002, 40, 2719-2728. 
https://doi.org/10.1002/pola.10301

30. Newkome, G. R.; Yao, Z.; Baker, G. R.; Gupta, V. K. J. Org. Chem. 1985, 50, 2003-2004. https://doi.org/10.1021/jo00211a052

31. Tomalia, D. A.; Baker, H.; Dewald, J.; Hall, M.; Kallos, G.; Martin, S.; Roeck, J.; Ryder, J.; Smith, P. Polym. J. 1985, 17, 117-132.

https://doi.org/10.1295/polymj.17.117

This paper is an open access article distributed under the terms of the Creative Commons Attribution (CC BY) license (http://creativecommons.org/licenses/by/4.0/) 